\title{
In Vitro Study of Biofilm Growth on Biologic Prosthetics
}

\author{
CHARLES BELLOWS ${ }^{1}$ and ALISON SMITH ${ }^{2}$ \\ ${ }^{1}$ University of New Mexico, Department of Surgery, Albuquerque, USA \\ ${ }^{1}$ Tulane University, New Orleans LA, USA
}

Submitted 14 November 2013, revised 12 July 2014, accepted 4 August 2014

\begin{abstract}
Biologic prosthetics are increasingly used for the repair of abdominal wall hernia defects but can become infected as a result of peri- or early post-operative bacterial contamination. Data evaluating biofilm formation on biologic prosthetics is lacking. The aim of this study was to investigate the influence of different biologic prosthetics on the growth behavior of two different bacterial species and their ability to form biofilms. Methicillin resistant Staphylococcus aureus (MRSA) or Pseudomonas aeruginosa were incubated on disks of two biologic prosthetics-human acellular dermis (ADM), and porcine small intestinal submucosa (SIS). The bacteria were allowed to attach to the prosthetics and propagate into mature biofilms for 24 hours at $37^{\circ} \mathrm{C}$. Images of biofilms were obtained using confocal microscopy and scanning electron microscopy (SEM). The number of viable cells and the biofilm biomass were quantified by colony forming units (CFUs) and crystal violet staining respectively. Analysis of variance was performed to compare the mean values for the different prosthetics. Each biologic matrix had a distinct surface characteristic. SEM visualized mature biofilms characterized by highly organized multi-cellular structures on surface of both biologic prosthetics. Quantification of bacterial growth over time showed that ADM had the lowest CFUs and biofilm biomass at 24 hours post-inoculation compared to SIS for both bacterial strains. MRSA and P. aeruginosa can form mature biofilms on biologic prosthetics but the relative abundance of the biofilm varies on different prosthetic constructs. Biologic material composition and manufacturing methods may influence bacterial adherence.
\end{abstract}

Key words: biofilm, biofilm biomass, biologic prosthetics, MRSA, P. aeruginosa

\section{Introduction}

Current medical evidence supports the use of synthetic prosthetic materials to repair ventral hernias as a means to reduce recurrence rates (Burger et al., 2004; Luijendijk et al., 2000). However, the routine use of this material for ventral hernia repair has resulted in several complications, most notably infection (Harth and Rosen, 2009). These prosthetic infections are a serious health problem and have emerged as one of the most challenging issues when considering ventral hernia repair. They are difficult to treat, lead to extended hospitalizations, additional surgical procedures, hospital readmissions, and time off from work. Infections can arise as a consequence of direct material contamination during implantation or the hematogenous spread of bacteria. These infections depend on the ability of the bacteria to attach to the material surface and the surface characteristics of the material influence this attachment (An and Friedman, 1998; Engelsman et al., 2008). Importantly, once attached, these microbes can begin to form a biofilm.
Staphylococcus aureus and Pseudomonas aeruginosa are two of the dominant causes of prosthetic infections. These opportunistic pathogens have the ability to attach to a substratum, accumulate biomass, and form a sessile community of cells encased within a self-produced matrix of extracellular polymeric substance, which is defined as a biofilm. This biofilm protects bacterial cells against the host's immune defenses and antimicrobial agents (Costerton et al., 1999; del Pozo and Patel, 2007; Hall-Stoodley and Stoodley, 2005; Zimmerli et al., 2004). The presence of a biofilm is increasingly being viewed as an important contributor to persistent prosthetic infections (Costerton et al., 2005 ).

In recent years, a new class of prosthetic materials made from animal and human products has emerged as a promising new material for the repair of ventral hernias, especially in contaminated surgical fields. These biologic prosthetics consist of an extracellular matrix, which has its cellular components removed. Unlike the synthetic prosthetics that are made from plastic, these biologic scaffold materials allow cellular in-growth (neovascularization) and site-specific tissue remodeling

\footnotetext{
* Corresponding author: Ch. Bellows, University of New Mexico, Department of Surgery, Albuquerque, USA; e-mail: cbellows@ salud.unm.edu
} 
into the original host's tissue (Badylak et al., 2001). Biologic prosthetics are believed to be more resistant to infections as the non-synthetic nature of the material allows for antibiotic diffusion. However, many different biologic prosthetics with diverse properties have been introduced on the market for clinical use and not all these materials are created equally (Bellows et al., 2011; Cornwell et al., 2009). Biologic prosthetics can differ in the tissue source, and manufacturing methods that decellularize and sterilize the material. These methods can have distinct effects on the surface characteristics of the resulting scaffold, thus affecting the ability of bacteria to attach and form a biofilm. The purpose of this study was to investigate biofilm production on biologic prosthetics with different composition and manufacturing properties using two different bacteria species in an in vitro model.

\section{Experimental}

\section{Materials and Methods}

Materials The biologic prosthetics used in this study were non-cross-linked human acellular dermis (ADM) (0.33-0.76 mm thickness; Alloderm, LifeCell, Branchburg, NJ), and non-cross-linked porcine small intestinal submucosa (SIS) (Surgisis Biodesign, Cook Surgical, West Lafayette, IN).

Scanning electron microscopy. Scanning electron microscopy (SEM) was used to visualize the surface characteristics of the biomaterial and the biofilm growth. For visualization of biofilms, the bacterial biofilms were grown on each biomaterial for 24 hours, following the methods described below, and SEM was performed. Briefly, following incubation, disks of each biologic prosthetic were gently rinsed with 1X PBS and removed using sterile forceps. A sample of each biomaterial was fixed in Karnovsky's fixative (Electron Microscopy Sciences, Hatfield, PA) for 30 minutes. After washing with cold cacodylate buffer, samples were post-fixed with 1\% osmium tetroxide (Sigma Aldrich, St. Louis, MO). Samples were then dehydrated in a series of ethanol solutions (30\% to $100 \%$ ) and were dried at the critical point. Specimens were mounted on aluminum stubs with conductive carbon cement, allowed to dry, and then coated with a thin layer of carbon (3-10 $\mathrm{nm}$ thickness). Samples were imaged using a Hitachi 4800 High-resolution SEM (Hitachi, Wallingford, CT). SEM experiments were carried out in duplicate for each strain tested, and representative images of biofilms were selected.

Bacterial strain and growth conditions. A clinical isolate of methicillin-resistant Staphylococcus aureus (MRSA) expressing GFP (provided by Dr. Alexander Horswill, Iowa University) and Pseudomonas aeru- ginosa strain PAO1 expressing GFP (provided by Dr. Lisa Morici, Tulane University) were cultured from a frozen glycerol stock and grown in Luria-Bertani (LB) broth overnight.

In vitro biofilm formation and experimental design. Biofilm formation was analyzed using a static biofilm system. Briefly, a sample of each biologic prosthetic was cut into $5 \mathrm{~mm}$ disks ( using a punch biopsy and rehydrated in phosphate buffered saline (PBS) for 30 minutes. The disks were then placed into individual wells of a 96 well tissue culture plate. Single bacterial strain biofilms were grown by incubating the disks for a maximum of 24 hours at $37^{\circ} \mathrm{C}$ in minimal essential medium alpha (MEMA) media supplemented with $10 \%$ fetal bovine serum (FBS) and L-Glutamine (Invitrogen, Carlsbad, CA) containing $1 \times 10^{7} \mathrm{CFUs} / \mathrm{mL}$ of $S$. aureus or P. aeruginosa respectively. This inoculum was chosen based on the observation that the concentrations of bacteria on normal skin flora are $10^{6-7} / \mathrm{cm}^{2}$ (Kloos, 1975). After 12 and 24 hours of incubation, biofilm-laden prosthetics were removed from each well, rinsed with $0.9 \% \mathrm{NaCl}$ and gently dried by minimal touch with gauze to remove planktonic bacteria. Prosthetic-associated biofilms were then analyzed for numbers of viable bacteria and biofilm biomass as described below. A single operator following a standardized protocol performed all experiments under sterile conditions. All experiments were performed in triplicate.

Extraction and quantification of biofilm. Prosthetic-associated biofilms were cultivated as described above. Biofilms were then disrupted by placing the sample in $0.5 \mathrm{~mL} 0.9 \% \mathrm{NaCl}$ containing $\alpha$-amylase from Aspergillus oryzae $(100 \mathrm{mg} / \mathrm{mL})$ (Sigma-Aldrich, St. Louis, MO) and incubated at room temperature for 90 minutes with gentle agitation. Serial dilutions $\left(10^{-1}\right.$ to $10^{-4}$ ) of the cell suspension were plated on LB agar and grown at $37^{\circ} \mathrm{C}$ in ambient air for 24 hours to calculate the numbers of viable bacteria as colony forming units (CFUs). This experiment was repeated three times with triplicates. Confocal microscopy confirmed nearly complete removal of the bacteria from the prosthetics.

In a separate experiment, the biomass was measured with crystal violet (Christensen et al., 1985 ). Briefly, biofilm-laden prosthetics were rinsed with PBS to remove any non-attached bacterial cells, fixed with 99\% methanol for $15 \mathrm{~min}$, air-dried and incubated for 15 min with $0.2 \%$ crystal violet (Fisher Chemical, Pittsburgh, PA). The prosthetics were washed thoroughly with distilled $\mathrm{H}_{2} \mathrm{O}$ and air-dried for 2 hours. Subsequently, the crystal violet absorbed by the biofilm was released by incubation with 33\% acetic acid for $15 \mathrm{~min}$ and the optical density was measured at $635 \mathrm{~nm}$ ( $\mu$ Quant microplate reader; Biotek Instruments, Winooski, VT). The average OD635 nm value was 
determined with three replicates. The background absorbance of the biologic prosthetics was measured and subtracted from the final data.

Statistical Analysis. Data represent the mean \pm standard error. Comparisons between the two groups were made using a Mann-Whitney U Test. Multiple comparison were performed using one-way Analysis of Variance (ANOVA) with Tukey's post hoc test if differences were found. All analyses were performed with Graphpad software (Graphpad, LaJolla, CA version 3.0). Statistical significance was defined as a $\mathrm{p}$ value less than 0.05 .

\section{Results}

Biologic prosthetics surface characterization. Scanning electron micrographs showed morphological and structural differences between the surfaces of the various biologic materials. Each biologic material had clefts and pores between the collagen fibers. One remarkably constant feature of ADM was the appearance of numerous ridges on the surface of the material

\section{ADM}
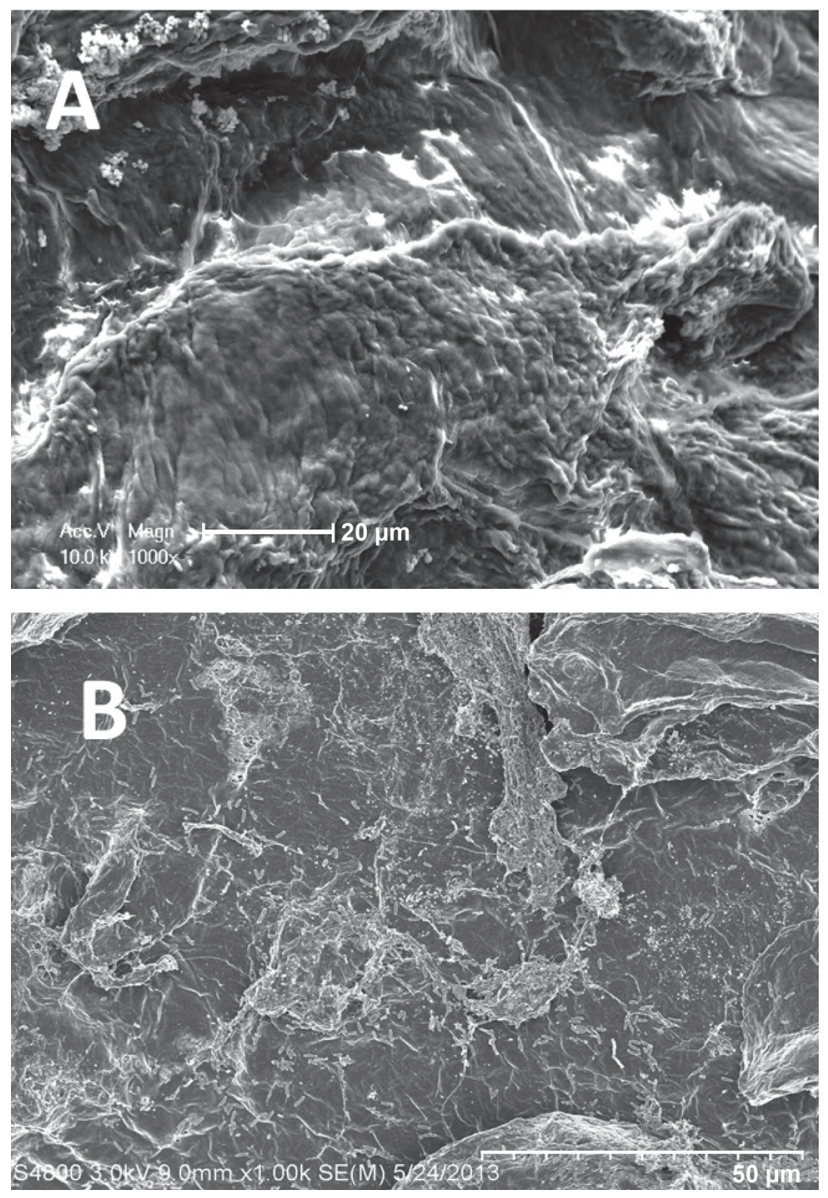

(Fig. 1A, B). By comparison, the surface of the SIS was relatively smooth characterized by tightly compacted clefts and pores (Fig. 1C, D).

SEM analysis of in vitro grown biofilm on biologic prosthetic surfaces. As seen in Figure 1, the extracellular polymeric substances (EPS) matrix of the biofilms was apparent in both MRSA and PAO1 biofilms and the different bacteria morphologies were clearly distinguishable under SEM. As demonstrated in Figure 1A, the surface of ADM demonstrated sparse bacterial colonization with small clumps of coccoid species evident on ridge-like structures with sparse extracellular products surrounding the cells aggregates. By comparison, colonization of the ADM surface by the PAO1 strains was seen as micro-colonies and the cell morphology was short thick rods interlaced with extracellular polymeric substance (Fig. 1B). In contrast, the surface of SIS demonstrated numerous cocci and micro-colonies that were widely distributed across the surface of the material within the biologic prosthesis (Fig. 1C). Figure 1D shows the scanning electron micrographs of the P. aeruginosa biofilm on the surface of SIS. Interestingly, the $\mathrm{PAO} 1$ species demonstrated profuse growth and dense
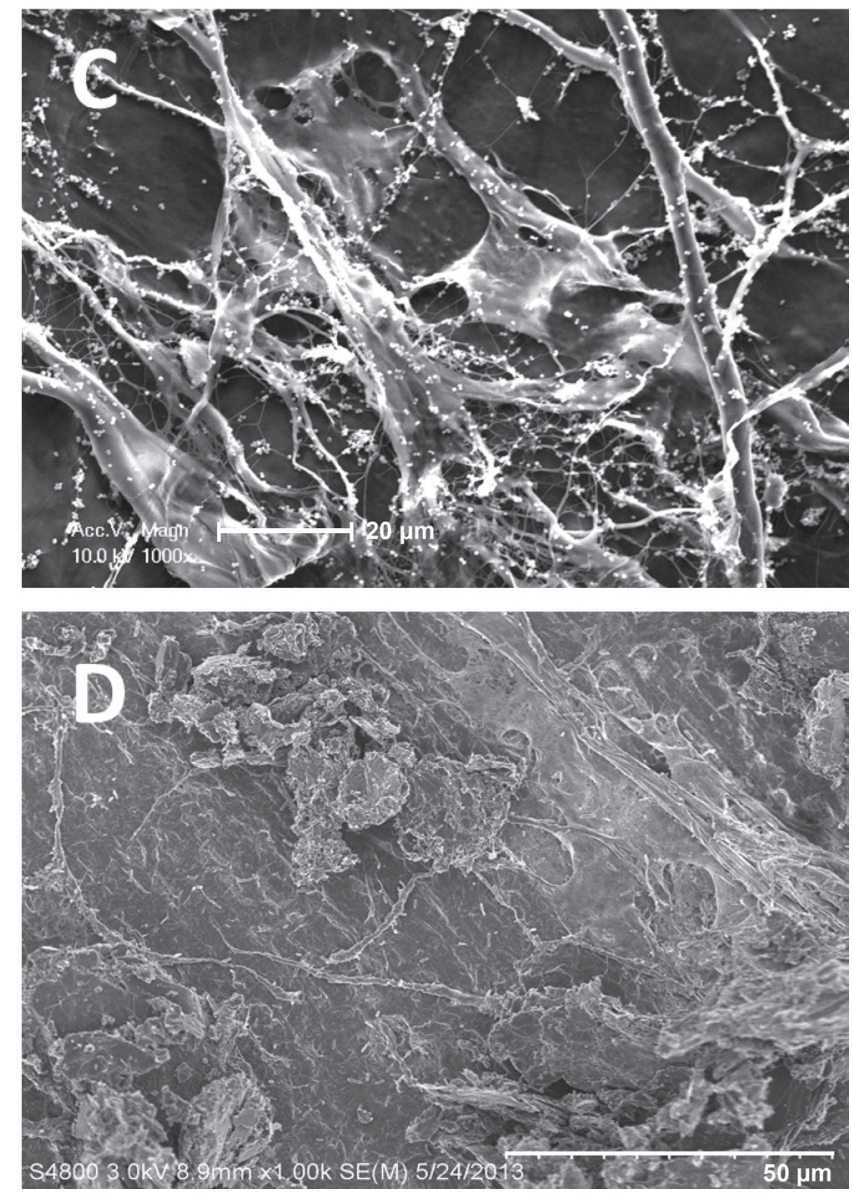

Fig. 1. Representative Scanning electron micrographs (SEM) images of methicillin resistant S. aureus (A) and P. aeruginosa (B) biofilms established on human acellular dermis (ADM) and methicillin resistant $S$. aureus (C) and P. aeruginosa (D) biofilms established on porcine small intestinal submucosa (SIS) following $24 \mathrm{~h}$ incubation at $37^{\circ} \mathrm{C}$. All images are $1000 \mathrm{X}$ magnification. 
colonization with recognizable thick extracellular products surrounding the cell aggregates (Fig. 1D).

In vitro growth curves of biofilm. The influence of the different biologic prosthetics on the ability to form biofilms and the growth behavior of these biofilm over time for the two different bacterial species were investigated. In general, we noted that both biologic prosthetics supported biofilm growth. However, more substantial biofilms in terms of bacterial growth (measured by CFU counts) and biofilm biomass (measured by crystal violet assay; OD635) were produced by PAO1 than by MRSA.

The biofilms produced by MRSA and PAO1 showed remarkable differences in growth patterns on the biologic prosthetics. For example, over the course of the 48-hour experiment, the number of viable MRSA cells (as measured in CFUs) significantly increased on the surface of SIS during the first 24 hours (Fig. 2A). By

\section{A}

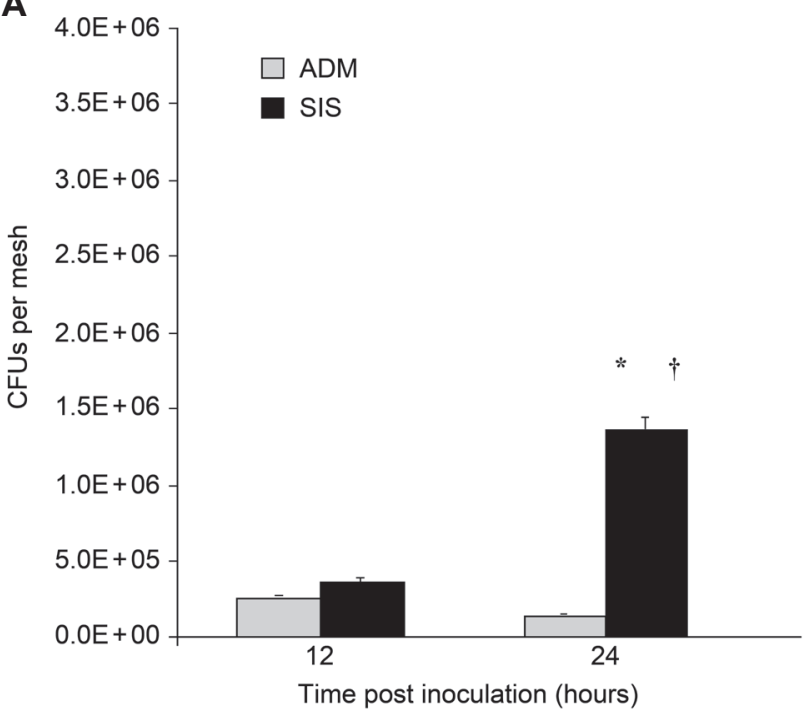

B

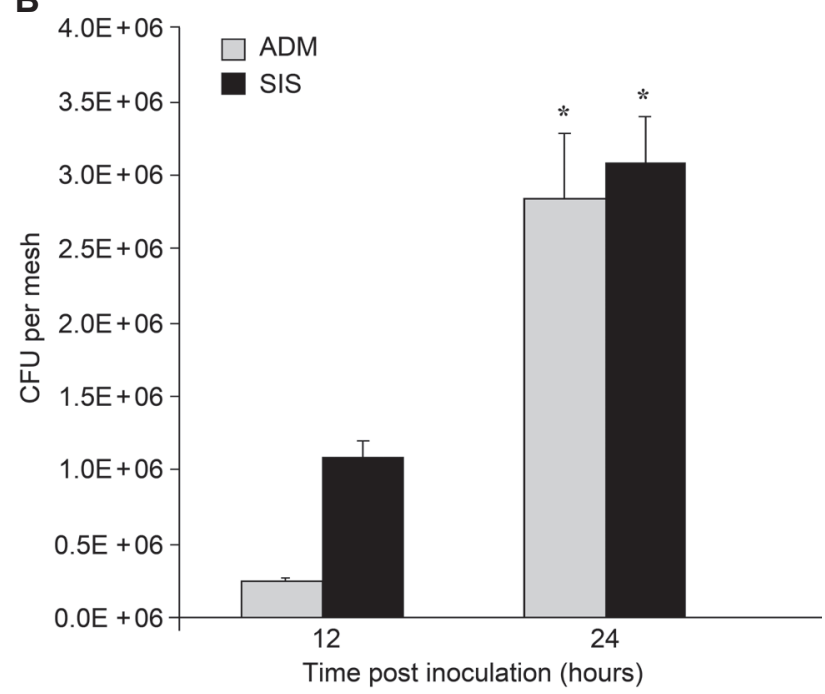

Fig. 2. Biofilm formation in vitro by S. aureus (A) or P. aeruginosa on human acellular dermis (ADM) or porcine small intestinal submucosa (SIS) as measured by CFUs over time $(n>6)$

${ }^{*} \mathrm{p}<0.05$ vs. $12 \mathrm{hr}$; ${ }^{* *} \mathrm{p}<0.05$ vs. $24 \mathrm{hr} ; \mathrm{t} \mathrm{p}<0.05$ vs. ADM

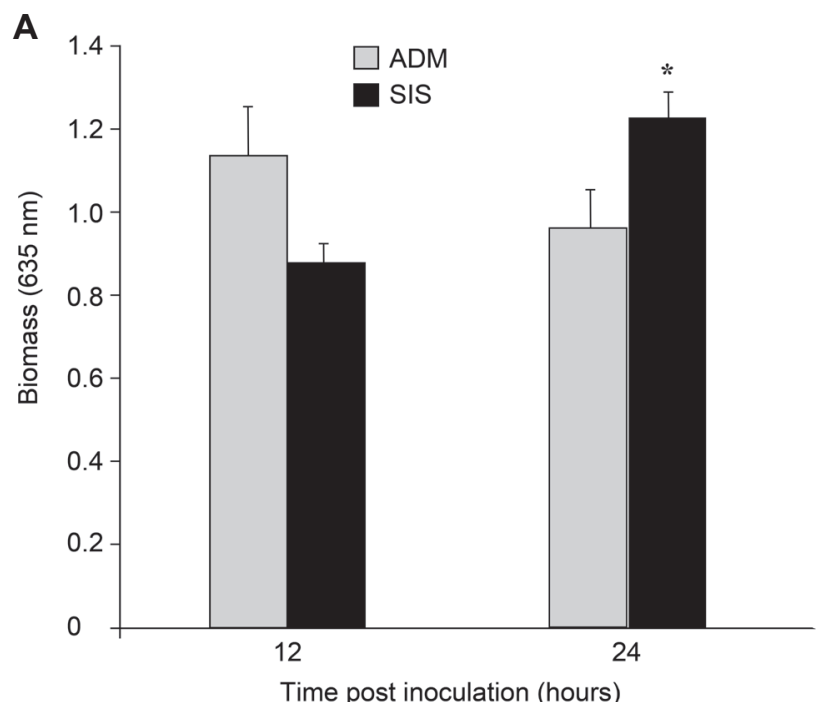

B

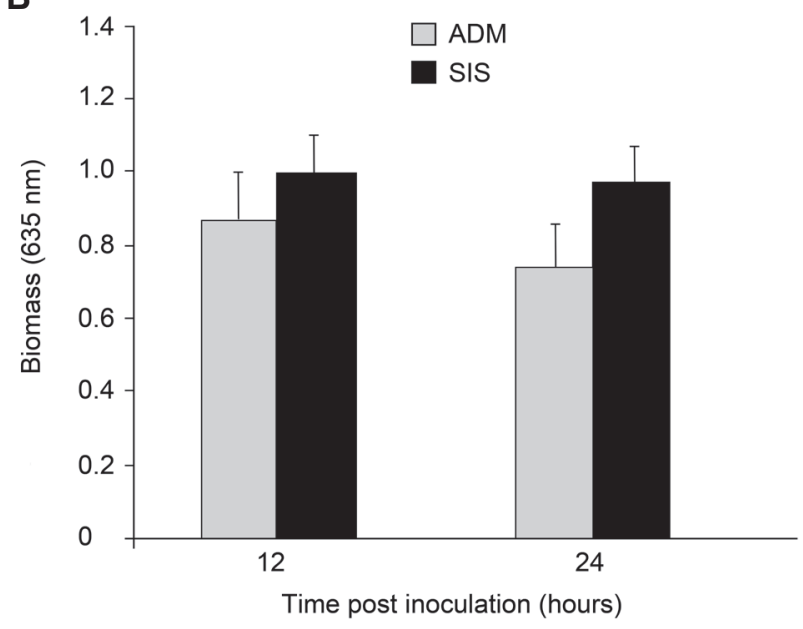

Fig. 3. Biofilm biomass formation in vitro by S.aureus (A) or P. aeruginosa (B) on human acellular dermis (ADM) or porcine small intestinal submucosa (SIS) as assessed by absorbance of crystal violet over time $(\mathrm{n} \geq 5)$ ${ }^{*} \mathrm{p}<0.05$ vs. $12 \mathrm{hr} ;{ }^{* *} \mathrm{p}<0.05$ vs. $24 \mathrm{hr}$

comparison, the number of live MRSA cells present on $\mathrm{ADM}$ was consistently low at every time point and significantly lower compared to SIS at 24 hours ( $\mathrm{p}<0.05$; Fig. 2A). For PAO1, we noted that both biologic prosthetics supported a steady increase in the number of CFUs over the first 24-hour period.

To further investigate the influence of the biologic prosthetics on biofilm formation, the biofilm biomass was determined at different time points post-inoculation (12 and 24 hours). As measured with crystal violet, the MRSA biofilm biomass reached its maximum at 12 hours after inoculation on the surface of ADM with a mean optical density (OD $635 \mathrm{~nm}$ ) of 1.15 (Fig. 3A). This value remained generally unchanged over the next 12 hours. By comparison, the MRSA biofilm biomass peaked at 24 hours after inoculation on the surface of SIS with mean optical density (OD $635 \mathrm{~nm}$ ) value of 1.23 (Fig. 3A). 


\section{Discussion}

The use of synthetic prosthetics for reinforcement has revolutionized the results of ventral hernia repair. However, due to concerns of infection many surgeons have turned to biologic prosthetics to repair complex abdominal wall defects especially in contaminated fields. However, this practice has resulted in a myriad of infectious complications related to the use of these biologic materials (Harth and Rosen, 2009). In fact, biomaterial-associated infections remain a major cause of failure of these prosthetic implants. Interestingly, data on the infection profile of the different biologic prosthetics in terms of susceptibility to bacterial adhesion and biofilm formation is sparse despite widespread use and significant associated costs (Bellows et al., 2013). Of the few studies in the literature, all have been conducted with reference to planktonic bacteria and less attention has been directed towards bacteria in the biofilm mode of growth. Understanding if the material type and/or the bacterial strain influences biofilm formation is essential for choosing the best prosthetic material.

The majority of these implant infections commonly involve opportunistic gram-positive (S.aureus) and gram-negative ( $P$. aeruginosa) pathogens (Engelsman et al., 2007; Falagas and Kasiakou, 2005; Mavros et al., 2011). Aggregates of these bacteria have a natural tendency to adhere to the surface of different prosthetic materials, and form biofilms, which prevent eradication of these organisms by conventional methods of killing such as antibiotics, and disinfection (Hall-Stoodley and Stoodley, 2009).

It is well-known that the initial bacterial cell attachment is critical for the formation of a bacterial biofilm. Several studies have shown that the roughness and material characteristics of the implant surface largely determine initial bacterial adherence (Engelsman et al., 2008; Aydinuraz et al., 2009; Halaweish et al., 2010; Stoodley et al., 2012). Therefore, using SEM for high-magnification imaging, we studied the surface morphology of the different biologic prosthetics. Interestingly, rough surfaces and niches, which can provide increased surface area and more contact points enabling adhesion of microorganisms, were observed on the surface of both materials. However, consistent with other authors, we also noted that the surface characteristics of each material were distinctly different depending on the organ or tissue from which the material was harvested (Brown et al., 2010).

To our knowledge, this in vitro study is the first that quantifies MRSA and P. aeruginosa biofilm formation on biologic prosthetics. Although previous studies (Engelsman et al., 2008; Aydinuraz et al., 2009; Halaweish et al., 2010; Stoodley et al., 2012) have defined similar models for synthetic prosthetics, an extensive literature search did not identify any studies that describe a method to quantify biofilms on biologic prosthetics. One of the major challenges in developing our model was effectively eluting the bacteria from the surface of the biologic prosthetics. Previous studies have used a combination of vortex and sonication to release bacteria from biofilms on synthetic prosthetics and to quantify it (Engelsman et al., 2008; Kobayashi et al., 2009). However, we found this method was ineffective on biologic prosthetics. Instead, we applied the results from a previous study by Craigen and colleagues who discovered that a commercially available $\alpha$-amylase could rapidly detach biofilms of $S$. aureus, inhibit biofilm formation and reduce and disassociate $S$.aureus cell-aggregates grown in liquid suspension (Craigen et al., 2011).

Overall, we have shown that among the biologic prosthetics tested, both were a suitable substratum for the production of biofilms containing viable, adherent cells over time under static conditions in vitro. Like others, we noted that the adhesion of $P$. aeruginosa was higher than S.aureus, leading to more robust biofilm formation (Borazjani, 2004; Reinis et al., 2011). However, we also observed that the biofilm development was highly variable on the surfaces of the different biologic prosthetics over time. In the MRSA group, the bacterial viability (as measured in CFUs) and the biofilm biomass production was more pronounced on the surface of SIS compared to ADM. In the Pseudomonas group, the bacterial viability was slightly higher on the surface of SIS but this did not reach statistical significance. However, it is noteworthy that the evident decrease in live bacteria from the surface of both biologic prosthetics measured by CFUs over time, did not equal the loss of biofilm biomass measured by crystal violet. This observation suggests a possible synergistic effect between the biologic prosthetics surfaces and P. aeruginosa biofilm biomass that promotes their persistence despite a loss of live bacteria. Interestingly, variances between biomass and viability measurements have also been shown with other medical devices (Henriques et al., 2006; Sule et al., 2009).

Taking the results from all assays together, we can clearly see distinct differences among the biologic prosthetic materials tested in terms of their ability to support bacterial viability and biofilm biomass. This may stem from the fact that not all the materials are created the same. There are significant differences in the process and manufacturing methods of the various biologic prosthetics harvested from various tissue sources (Bellows et al., 2011; Cornwell et al., 2009). These methods can significantly impact the materials structure and surface topographies, which is used as substrata for bacterial attachment (Cornwell et al., 2009; Aydinuraz et al., 2009; Gilbert et al., 2006).

There are several limitations of our study. We only studied two biologic prosthetics, but there are currently over a dozen commercially available biologic prosthetics for use in ventral hernia repair. Moreover, we 
only conducted an in vitro analysis. These studies will need to be repeated in other relevant in vivo models to confirm these results.

In conclusion, before now little was known about the ability to form biofilms on biologic prosthetics. We observed that biofilm formation on biologic prosthetics was both material and species dependent. The type of material and/or the processing technique may influence the attachment and growth of certain microorganisms. This result could have significant implications for the selection of biologic prosthetics by surgeons in clinical settings, such as in contaminated surgical fields.

\section{Acknowledgements}

We acknowledge Dr. Alexander Horswill of the University of Iowa for providing the GFP-labeled MRSA strain. We would like to thank Dr. Jiabo He of Tulane University for his assistance in obtaining the scanning electron microscopy images. We would like to thank Dr. Kerstin Honer zu Bentrup for her assistance with microscopy and edits of the manuscript.

\section{Conflict of interest}

Charles Bellows and Alison Smith declare that there is no conflict of interest.

\section{Literature}

An Y. and R. Friedman. 1998. Concise review of mechanisms of bacterial adhesion to biomaterial surfaces. J. Biomed. Mater. Res. 43: 338-348.

Aydinuraz K., C. Agalar, F. Agalar, S. Ceken, N. Duruyurek and T. Vural. 2009. In vitro S. epidermidis and S. aureus adherence to composite and lightweight polypropylene grafts. J. Surg. Res. 157: e79-86. Badylak S., K. Kokini, B. Tullius and Whitson B. 2001. Strength over time of a resorbable bioscaffold for body wall repair in a dog model. J. Surg. Res. 99: 282-287.

Bellows C., A. Smith, J. Hodde and M. Hiles. 2011. Tissue engineering in abdominal wall surgery. Minerva Chir. 66: 129-143.

Bellows C., A.Smith, J. Malsbury and W. Helton. 2013 Repair of incisional hernias with biological prosthesis: a systematic review of current evidence. Am. J Surg. 205: 85-101.

Borazjani R. 2004. Relative primary adhesion of Pseudomonas aeruginosa, Serratia marcescens and Staphylococcus aureus to HEMAtype contact lenses and an extended wear silicone hydrogel contact lens of high oxygen permeability Cont. Lens Anterior Eye. 27: 3-8. Brown B., C. Barnes, R. Kasick, R. Michel, T. Gilbert, D. BeerStolz, D. Castner, B. Ratner and S. Badylak. 2010. Surface characterization of extracellular matrix scaffolds. Biomaterials 31: 428-437. Burger J., R. Luijendijk, W. Hop, J. Halm, E. Verdaasdonk and J. Jeekel. 2004. Long-term follow-up of a randomized controlled trial of suture versus mesh repair of incisional hernia. Ann. Surg. 240: 578-583.

Christensen G., W. Simpson, J. Younger, L. Baddour, F. Barrett, D. Melton and E. Beachey. 1985. Adherence of coagulase-negative staphylococci to plastic tissue culture plates: a quantitative model for the adherence of staphylococci to medical devices. J. Clin. Microbiol. 22: 996-1006.

Cornwell K., A. Landsman and K. James. 2009 Extracellular matrix biomaterials for soft tissue repair. Clin. Podiatr. Med. Surg. 26: 507-523.

Costerton J., L. Montanaro and C. Arciola. 2005. Biofilm in implant infections: its production and regulation. Int. J. Artif. Organs. 28: 1062-1068.
Costerton J., P. Stewart and E. Greenberg. 1999. Bacterial biofilms: a common cause of persistent infections. Science 284: 1318-1322. Craigen B., A.Dashiff and D. Kadouri. 2011. The use of commercially available alpha-amylase compounds to inhibit and remove Staphylococcus aureus biofilms. Open Microbiol. J. 5: 21-31.

del Pozo J. and R. Patel. 2007. The challenge of treating biofilmassociated bacterial infections. Clin. Pharmacol. Ther. 82: 204-209. Engelsman A., H. van der Mei, H. Busscher and R. Ploeg. 2008. Morphological aspects of surgical meshes as a risk factor for bacterial colonization. Br. J. Surg. 95: 1051-1059.

Engelsman A., H. Van der Mei, R. Ploeg and H. Busscher. 2007. The phenomenon of infection with abdominal wall reconstruction. Biomaterials 28: 2314-2327.

Falagas M. and S. Kasiakou. 2005. Mesh-related infections after hernia repair surgery. Clin. Microbiol. Infect. 11:3-8.

Gilbert T., T. Sellaro and S. Badylak. 2006. Decellularization of tissues and organs. Biomaterials 27: 3675-3683.

Hall-Stoodley L. and P. Stoodley. 2005 Biofilm formation and dispersal and the transmission of human pathogens. Trends Microbiol. 13: 7-10.

Hall-Stoodley L.and P.Stoodley. 2009 Evolving concepts in biofilm infections. Cell. Microbiol. 11: 1034-1043.

Halaweish I., K. Harth, A.Broome, G. Voskerician, M. Jacobs and M. Rosen. 2010. Novel in vitro model for assessing susceptibility of synthetic hernia repair meshes to Staphylococcus aureus infection using green fluorescent protein-labeled bacteria and modern imaging techniques. Surg. Infect. (Larchmt) 11: 449-454.

Harth K. and M. Rosen. 2009. Major complications associated with xenograft biologic mesh implantation in abdominal wall reconstruction. Surg. Innov. 16: 324-329.

Henriques M., J. Azeredo J. and R. Oliveira. 2006. Candida albicans and Candida dubliniensis: comparison of biofilm formation in terms of biomass and activity. Br. J. Biomed. Sci. 63: 5-11.

Kloos W. and M. Musselwhite. 1975. Distribution and persistence of Staphylococcus and Micrococcus species and other aerobic bacteria on human skin. Appl. Microbiol. 30: 381-385.

Kobayashi H., M. Oethinger, M. Tuohy, G. Procop and T. Bauer. 2009. Improved detection of biofilm-formative bacteria by vortexing and sonication: a pilot study. Clin. Orthop. Relat. Res. 467: 1360-1364. Luijendijk R., W. Hop, M. van den Tol, D. de Lange, M. Braaksma, J. IJzermans, R. Boelhouwer, B. de Vries, M. Salu, J. Wereldsma, C. Bruijninckx and J. Jeekel. 2000. A comparison of suture repair with mesh repair for incisional hernia. N. Engl. J. Med. 343: 392-398. Mavros M., S. Athanasiou, V. Alexiou, P. Mitsikostas, G. Peppas and M. Falagas. 2011. Risk factors for mesh-related infections after hernia repair surgery: a meta-analysis of cohort studies. World J. Surg. 35: 2389-2398.

Stoodley P., S. Sidhu, L. Nistico, M. Mather, A. Boucek, L. HallStoodley and S. Kathju. 2012. Kinetics and morphology of polymicrobial biofilm formation on polypropylene mesh. FEMS Immunol. Med. Microbiol. 65: 283-290.

Reinis A., M. Pilmane, A.Stunda, J. Vētra, J. Kroiča, D. Rostoka, G. Salms, A. Vostroilovs, A. Dons and L. Bērziña-Cimdiña. 2011. An in vitro and in vivo study on the intensity of adhesion and colonization by Staphylococcus epidermidis and Pseudomonas aeruginosa on originally synthesized biomaterials with different chemical composition and modified surfaces and their effect on expression of TNF- $\alpha, \beta$-defensin 2 and IL-10 in tissues. Medicina (Kaunas), 45: 560-565.

Sule P., T. Wadhawan, N. Carr, S. Horne, A. Wolfe and B. Prüss. 2009. A combination of assays reveals biomass differences in biofilms formed by Escherichia coli mutants. Lett. Appl. Microbiol. 49, 299-304.

Zimmerli W., A.Trampuz and P. Ochsner. 2004. Prosthetic-joint infections. N. Engl. J. Med. 351: 1645-1654. 